\title{
THE EVOLUTION OF NON-ADIABATIC PRESSURE PERTURBATIONS DURING MULTI-FIELD INFLATION
}

\author{
IAN HUSTON \\ Astronomy Unit, School of Physics and Astronomy, Queen Mary University of London, \\ Mile End Road, London, E1 4NS, United Kingdom \\ ADAM J. CHRISTOPHERSON \\ School of Physics and Astronomy, University of Nottingham, \\ University Park, Nottingham, NG7 2RD, United Kingdom
}

\begin{abstract}
Non-adiabatic pressure perturbations naturally occur in models of inflation consisting of more than one scalar field. The amount of non-adiabatic pressure present at the end of inflation can have observational consequences through changes in the curvature perturbation, the generation of vorticity and subsequently the sourcing of B-mode polarisation. In this work, based on a presentation at the 13th Marcel Grossmann Meeting, we give a very brief overview of non-adiabatic pressure perturbations in multi-field inflationary models and describe our recent calculation of the spectrum of isocurvature perturbations generated at the end of inflation for different models which have two scalar fields.
\end{abstract}

Keywords: cosmological perturbation theory, isocurvature, non-adiabatic pressure

\section{Introduction}

In part due to the possibility of observable non-Gaussian signals, the study of inflationary scenarios has recently focussed on models with more than one operative scalar field. The extra degrees of freedom present in these physical systems support isocurvature or non-adiabatic pressure modes in addition to the usual adiabatic mode.

Cosmological perturbation theory is a well-established technique for analysing the inflationary perturbations which allows us to numerically compute the nonadiabatic pressure throughout the inflationary era. (See Ref. 11 and references therein for a comprehensive introduction to perturbation theory.)

One reason for considering the non-adiabatic pressure perturbation produced towards the end of inflation is that non-adiabatic pressure perturbations can source vorticity at second order in perturbation theory ${ }^{[2}$ This vorticity is expected to have some effect on the CMB, as vector perturbations can source B-mode polarisation.

In addition the presence of non-adiabatic pressure at the end of inflation can affect the predictive power of an inflationary model. The conservation of the curvature perturbation at large scales requires the absence of non-adiabatic pressure.$^{1}$ To connect inflationary results with CMB data in the presence of non-adiabatic modes then requires that the full evolution through reheating and radiation domination is known. 


\section{Non-adiabatic pressure in multi-field inflation}

By considering scalar perturbations at linear order to the energy-momentum tensor and a flat Friedmann-Robertson-Walker metric we can find the equations of motion of the system using the perturbed Einstein equations. Here we consider perturbations in the uniform curvature gauge where the scalar curvature perturbation is identically zero. In the following we show results for a system of two scalar fields $\varphi$ and $\chi$, but the results are generalisable to many field systems and the numerical code discussed below handles the full multi-field case.

In general a fluid, in this case the combined scalar field fluid, is not purely adiabatic. The full pressure perturbation $\delta P$ can then be split as

$$
\delta P=\delta P_{\mathrm{nad}}+c_{\mathrm{s}}^{2} \delta \rho,
$$

where $\delta P_{\text {nad }}$ is the non-adiabatic pressure perturbation, $c_{\mathrm{s}}^{2}=\dot{P} / \dot{\rho}$ is the adiabatic sound speed for the fluid and $\delta \rho$ is the energy density perturbation.

For the case of two scalar fields with a combined potential $V(\varphi, \chi)$, the nonadiabatic pressure perturbation at linear order is given by

$$
\begin{aligned}
\delta P_{\mathrm{nad}}= & \frac{8 \pi G}{3 H^{2}}\left(V_{, \varphi} \dot{\varphi}+V_{, \chi} \dot{\chi}\right)(\dot{\varphi} \delta \varphi+\dot{\chi} \delta \chi)-2\left(V_{, \varphi} \delta \varphi+V_{, \chi} \delta \chi\right) \\
& -\frac{2}{3 H} \frac{\left(V_{, \varphi} \dot{\varphi}+V_{, \chi} \dot{\chi}\right)}{\left(\dot{\varphi}^{2}+\dot{\chi}^{2}\right)}\left[\dot{\varphi} \dot{\phi} \dot{\varphi}+\dot{\chi} \dot{\chi} \dot{\chi}+V_{, \varphi} \delta \varphi+V_{, \chi} \delta \chi\right],
\end{aligned}
$$

where $V_{, \varphi}=\partial V / \partial \varphi$ and $\delta \varphi$ and $\delta \chi$ are the linear perturbations in the $\varphi$ and $\chi$ fields.

\section{Discussion}

In Ref. 3 we numerically calculated the non-adiabatic pressure for a number of interesting inflationary models. We used the Pyflation package for Python, an open source numerical package created by one of the authors and described in Refs. 4 - 6 . The numerical system evolves the background and first order perturbations of the scalar fields from deep inside the cosmological horizon until the end of inflation. For $n$ scalar fields the full $n \times n$ matrix of mode amplitudes for the quantum creation operators are evolved and only one run is necessary. This is in contrast to other approaches in which $n$ separate runs are required, with the initial condition for only one of the field perturbations being non-zero in each run.

We examined the production of non-adiabatic pressure in Ref. 3 for three potentials widely considered in the literature: a double quadratic model $\frac{1}{2} m_{\varphi}^{2} \varphi^{2}+\frac{1}{2} m_{\chi}^{2} \chi^{2}$, a quartic model $\Lambda^{4}\left[\left(1-\chi^{2} / v^{2}\right)^{2}+\varphi^{2} / \mu^{2}+2 \varphi^{2} \chi^{2} /\left(\varphi_{\mathrm{c}}^{2} v^{2}\right)\right]$, and a product exponential model $V=V_{0} \varphi^{2} e^{-\lambda \chi^{2}}$. The results for the non-adiabatic pressure of the product exponential model are shown in Fig. 1. In calculating these results we have set $\lambda=0.05 / M_{\mathrm{pl}}^{2}$ and normalized the power spectrum to the WMAP value by setting $V_{0}=5.37 \times 10^{-13} M_{\mathrm{pl}}^{2}$. The initial field values are $\varphi_{0}=18 M_{\mathrm{pl}}$ and $\chi_{0}=0.001 M_{\mathrm{pl}}$.

These results are for one particular wavemode, the WMAP pivot scale, and compare the evolution of the full pressure perturbation with the non-adiabatic part over 
the course of the inflationary expansion. For this model the amount of isocurvature present at the end of inflation is significant enough to warrant careful examination of the evolution after inflation, especially during the reheating phase. As such any prediction of the statistics of curvature perturbations given at the end of inflation is subject to modification during a reheating phase and radiation domination. We will explore the role of non-adiabatic pressure and the evolution of curvature perturbations during reheating in a future publication. ${ }^{7}$

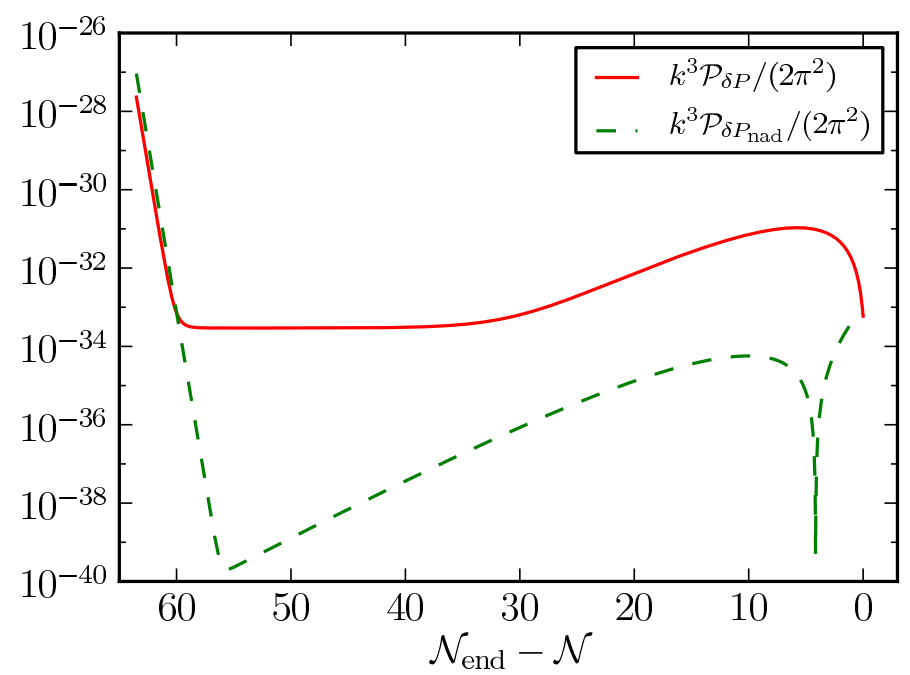

Fig. 1. A comparison of the scaled power spectra of $\delta P$ (red straight line) and $\delta P_{\text {nad }}$ (green dashed line) for the product exponential potential at the WMAP pivot scale. The $\mathrm{x}$-axis denotes the number of e-foldings before the end of inflation, $\mathcal{N}_{\text {end }}$.

\section{Acknowledgements}

IH is supported by the STFC under Grant ST/G002150/1, and AJC is funded by the Sir Norman Lockyer Fellowship of the Royal Astronomical Society.

\section{References}

1. K. A. Malik and D. Wands, Phys. Rept. 475, 1 (2009).

2. A. J. Christopherson, K. A. Malik and D. R. Matravers, Phys. Rev. D79, p. 123523 (2009).

3. I. Huston and A. J. Christopherson, Phys.Rev. D85, p. 063507 (2012).

4. I. Huston and K. A. Malik, JCAP 1110, p. 029 (2011).

5. I. Huston and K. A. Malik, JCAP 0909, p. 019 (2009).

6. I. Huston, Pyflation: Cosmological perturbations for Python http://pyflation.ianhuston.net, (2011-).

7. I. Huston and A. J. Christopherson, in preparation (2012). 